\title{
A fidget spinner device for UTI diagnosis
}

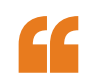

The presence of

bacteria in the

urine could be

detected within

50 minutes,

meaning that

appropriate anti-

biotics could be

quickly offered
A novel device based on the popular fidget spinner toy has been shown to be effective for point-of-care (POC) diagnosis of UTI and could be useful in low-resource settings. The study has been published in Nature Biomedical Engineering.

UTI is a common problem worldwide and in modern laboratories is usually diagnosed using culture and sensitivity testing to identify and appropriately target the pathogen. However, in low-resource settings, such as rural areas, these diagnostic tests are often not available; thus, diagnosis is instead based on symptoms alone and patients are prescribed broad-spectrum antibiotics, with associated sequelae including antibiotic resistance and harm to the patients' microbiome. POC testing (POCT) devices are, therefore, in demand to reduce the need for laboratory diagnosis and improve accuracy of antibiotic prescriptions.

Based on this necessity, Michael and colleagues developed a diagnostic fidget spinner (Dx-FS) that uses the centrifugal force generated by spinning the device to act like a centrifuge. Urine from a patient suspected to have a UTI is applied to the sample chamber and the device is spun. Spinning the Dx-FS causes bacteria present in the urine sample

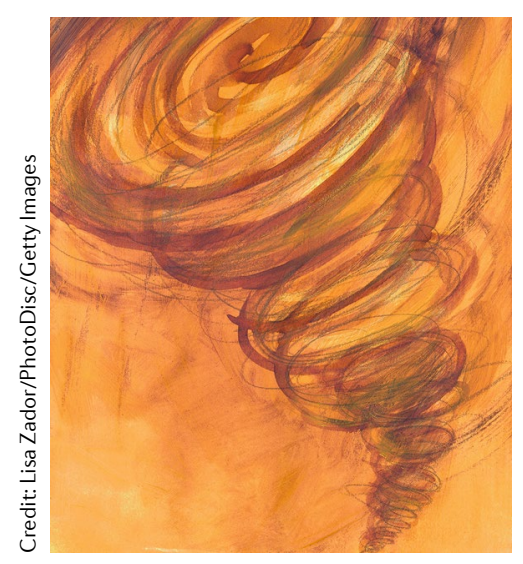

to become lodged on a nitrocellulose membrane, where they can then be detected with the naked eye using colorimetry.

The device is spun by hand to create centrifugal force but has a relatively low spinning speed. The speed of spinning is operator dependent and also slows over the course of a minute after it is pushed. These variabilities were accommodated in the Dx-FS by engineering the microfluidics using fluid-assisted separation technology (FAST), which maintains the filtration efficiency of the device and also enables uniform spreading of any bacterial cells present over the membrane. The operator-dependent aspects of the Dx-FS function were tested by asking operators of various ages (19-45 years) to spin the device. Large variations in spin speed were noted, but integration of FAST into the early model meant that only one or two manual spins (taking 1-3 minutes) were needed to distribute the $1 \mathrm{ml}$ sample volume, regardless of the operator.

In order to make Dx-FS as straightforward and useful as possible, the operation of the device was simplified to include just three steps: sample injection (administration of $1 \mathrm{ml}$ urine using a plastic pipette through the inlet hole), bacterial enrichment (spinning of the device until the sample is filtered), and detection (removal of FAST solution and addition of detection solution and spinning until the detection solution is in the membrane chamber, after which the colour change is measured and translated into bacterial load). The colour change was measured using an integrated WST- 8 assay. This assay uses an electron mediator to receive electrons from bacterial cells and transfer them to the WST-8, causing a colour change to an orange formazan dye, which can be detected with the naked eye and compared to a colour chart to levels of viable bacteria.

Having ensured that the Dx-FS complied with WHO-assured standards, the authors went on to test the device in the field in Tiruchirappalli, India, an area where most patients come from rural areas and prescription of antibiotics for UTI is generally based on symptoms rather than a clinical diagnosis. In this study, samples were collected from 39 patients and tested using the Dx-FS and conventional urinalysis and culturing in a hospital laboratory. The results achieved using culture and Dx-FS were comparable and independent of patient gender and age, and of bacterial strain. The presence of bacteria in the urine could be detected within 50 minutes, meaning that appropriate antibiotics could be quickly offered.

The Dx-FS was further refined by addition of an antimicrobial susceptibility test (AST) to develop the Fidget-AST. On-device AST for Escherichia coli (which causes $\sim 80 \%$ of UTIs) was able to discriminate samples that were resistant or susceptible to ciprofloxacin or cefazolin. Although Fidget-AST was only tested for E. coli in this study, future work could test different causative bacteria.

For Dx-FS to be usable as a POCT platform in resource-poor areas, it must be possible for it to be used without laboratory facilities, expert operators or power supplies and it must be cost effective. As the material cost of a single Dx-FS device, which can test 2 samples, is estimated at just US $\$ 0.48$ (which can be further minimized by scaling), the device has considerable potential. "Overall, the results demonstrate that the Dx-FS is a disruptive technology that meets the need for UTI management using POCT devices, especially in resource-limited settings," the authors conclude.

Annette Fenner

ORIGINAL ARTICLE Michael, I. et al. A fidget spinner for the point-of-care diagnosis of urinary tract infection. Nat. Biomed. Eng. 4, 591-600 (2020) 